\title{
Linx
}

Revue des linguistes de l'université Paris X Nanterre

$57 \mid 2007$

Études de syntaxe : français parlé, français hors de

France, créoles

\section{Valeur sémantique et comportement syntaxique de l'infinitif substitut dans quelques corpus oraux}

\section{Anika Falkert}

\section{(2) OpenEdition}

\section{Journals}

Édition électronique

URL : http://journals.openedition.org/linx/76

DOI : $10.4000 /$ linx.76

ISSN : 2118-9692

Éditeur

Presses universitaires de Paris Nanterre

Édition imprimée

Date de publication : 1 décembre 2007

Pagination : 69-78

ISSN : 0246-8743

Référence électronique

Anika Falkert, « Valeur sémantique et comportement syntaxique de l'infinitif substitut dans quelques corpus oraux », Linx [En ligne], 57 | 2007, mis en ligne le 15 février 2011, consulté le 15 mai 2020. URL http://journals.openedition.org/linx/76 ; DOI : https://doi.org/10.4000/linx.76 


\title{
Valeur sémantique et comportement syntaxique de l'infinitif substitut dans quelques corpus oraux
}

\author{
Anika Falkert \\ Université d'Avignon et EA 4277
}

\section{Introduction}

Si le français oral et le français écrit ne possèdent pas deux «grammaires » différentes, les recherches visant à une description syntaxique de l'oral ne cessent de souligner que seule une approche globale peut aboutir à une vision plus claire du fonctionnement des échanges ${ }^{1}$. Ces analyses devraient envisager les constructions du point de vue de leur comportement syntaxique, de leur valeur sémantique et/ou pragmatique et des facteurs prosodiques (structures intonatives, pauses, etc.). Sans postuler l'existence d'une «syntaxe de l'oral », il importe d'appréhender la construction du sens à l'oral comme une construction dans le temps, la linéarité imposée déterminant au moins partiellement les structures syntaxiques. La remise en question du concept de la phrase (v. Le Goffic 2005) et la distinction entre micro- et macrosyntaxe ${ }^{2}$ n'ont été qu'un premier pas vers une meilleure compréhension de l'oralité.

\footnotetext{
${ }^{1}$ V. par exemple Falkert (2006) à propos du rôle de la prosodie dans l'analyse du marqueur discursif ça fait que.

${ }^{2}$ V. Berrendonner (1990) et Blanche-Benveniste (1990).
} 
L'observation des phénomènes syntaxiques des variétés périphériques ayant évolué dans un contexte d'oralité où la pression de la norme s'avère faible, sinon absente, présente un intérêt particulier. Dans le cadre de cette étude, nous nous pencherons sur un aspect qui illustre la «tendance à l'invariance, qui atteint les accords (genre et nombre) et les concordances (temps et modes) » (Gadet 1989: 114) et qui caractérise les évolutions dans le français parlé, que ce soit en français familier et/ou populaire ou dans les variétés hors de France.

\section{L'infinitif substitut est décrit par Remacle (1952 : 142) comme un infinitif}

«qui suit toujours une forme verbale complète, se présente lui-même comme une forme verbale aussi nue, aussi réduite que possible ; on dirait que le locuteur ne trouve plus nécessaire de recourir une seconde fois à tous les moyens ».

Selon Grevisse (2008), «dans le français parlé de Wallonie et du Nord de la France, il est assez fréquent qu'un infinitif soit coordonné à un verbe conjugué dont il devrait avoir le mode, le temps et la personne », comme dans l'exemple suivant :

[1] Pourquoi que t'apportes pas une balance et me laisser te peser (Grevisse $2008:$ : 901e).

C'est aussi le cas dans les variétés de français d'Amérique du Nord, comme par exemple dans le parler acadien des Îles-de-la-Madeleine :

[2] mais: ce qui est le FUN ici c'est./ c'est que le monde est sociable on parle à tout le monde tout le monde se connaît on se promène dans la rue HELLO ((rit)) salut. pis: en ville. Montréal Québec tu te promènerais pas dans le rue pis dire salut au monde pace qu'i vont dire c'est quoi ((rit)) (IdlM, BA03, 36-42).

Brasseur (1998) mentionne des attestations de cette construction en Suisse et en lorrain roman ainsi que dans le dialecte anglo-normand de l'Île de Sercq. Les études de Brasseur (1998) et Neumann-Holzschuh (2003) qui visent à éclaircir les emplois de cette construction mettent en avant le rapport entre l'ellipse du pronom personnel ${ }^{3}$ et la multiplication des formes invariables dans les variétés périphériques. Par ailleurs, les deux chercheurs font ressortir un rapprochement éventuel avec les constructions verbales sérielles que l'on trouve dans les créoles français ${ }^{4}$.

L'objectif de cet article n'est pas de poursuivre cette réflexion, mais d'analyser en détail des occurrences de l'infinitif substitut dans plusieurs corpus du français parlé.

\footnotetext{
${ }^{3}$ Exemple : «I 'tait pas pêcheur. faisait/ travaillait sur l'eau là mais : i a fait' plein de métiers là » (IdlM, BA02, 43-44).

4 «Constatons, au passage, que quelques-uns de ces énoncés et de ceux qui suivent peuvent évoquer les constructions verbales sérielles des langues créoles. Nous rappellerons, à cet égard, que TerreNeuve partage avec les créoles plusieurs éléments d'ordre historique et linguistique [...]. La colonisation de la plupart des îles de l'Océan Indien et des Antilles s'est effectuée, globalement, à la même époque que la colonisation acadienne. [...] Par ailleurs, les créoles et les variétés du français d'Amérique du Nord, notamment les isolats de Nouvelle-Écosse et de Terre-Neuve, ont un ancrage particulièrement évident dans le français populaire et une transmission presque exclusivement orale » (Brasseur 1998 : 86).
} 
Valeur sémantique et comportement syntaxique de l'infinitif substitut dans quelques corpus oraux

Nos hypothèses s'appuient sur les exemples tirés de quatre corpus du français acadien $^{5}$, deux corpus du français cadien ${ }^{6}$, un corpus du français québécois ${ }^{7}$ et d'un corpus du français hexagonal ${ }^{8}$. Pour ce qui est du français québécois, nous citerons également quelques exemples de Seutin (1975). Étant donné que nous n'avons pas accès aux enregistrements des différents corpus, l'analyse des facteurs suprasegmentaux se limitera à la prise en compte des pauses, clairement indiquées dans les transcriptions. Nous nous proposons d'examiner les différentes fonctions que l'infinitif substitut peut revêtir et de déceler dans quelle mesure l'emploi de cette construction modifie le rapport entre la dépendance sémantique et la dépendance syntaxique d’un énoncé.

Les données diachroniques faisant mention de l'infinitif substitut sont très lacunaires. Par ailleurs, un rapprochement avec l'infinitif de narration ${ }^{9}$ semble improbable même si celui-ci exprime une relation de coordination, explicite ou implicite, et « a pu autrefois être construit sans préposition»(Grevisse 2008: \ 901), comme dans l'exemple suivant :

[3] Lors Oudart se revestir [...]; chacun rire, tous se préparer (Rabelais, cité dans Grevisse $2008:$ \901c).

En effet, l'infinitif substitut s'appuie systématiquement sur une forme verbale conjuguée en début de la séquence, ce qui n'est pas le cas dans l'exemple [3].

Compte tenu des attestations très diverses dans les corpus, une différenciation des emplois selon la valeur sémantique véhiculée par cette construction s'impose. On distinguera tout d'abord l'infinitif substitut dans le récit où il marque soit une suite d'actions ou d'événements qui se succèdent, soit des routines ou des actions récurrentes.

\footnotetext{
${ }^{5}$ Nouveau-Brunswick : Arrighi (désormais NB-A), Wiesmath $(\mathrm{NB}-\mathrm{W}$ ) ; Terre-Neuve : Brasseur (TN) ; Îles-de-la-Madeleine : Falkert (IdlM).

${ }^{6}$ Stäbler (Lou - S), Valdman et al. (Lou - LFDP).

7 Il s'agit du corpus «Estrie », qui comprend des enquêtes orales effectuées en 1971-1972 dans la région de Sherbrooke sous la direction de Normand Beauchemin et Pierre Martel et publiées sous le titre d'Échantillons de textes libres (v. références bibliographiques).

${ }^{8}$ Corpus Beeching, disponible en ligne : http://www.uwe.ac.uk/hlss/llas/iclru/corpus.pdf

${ }^{9}$ L'infinitif de narration ou infinitif historique est attesté dès l'ancien français et considéré comme familier au XVII siècle (v. Grevisse 2008 : \901c). «Cet emploi en proposition indépendante de l'infinitif avec de, précédé le plus souvent d'un nom ou d'un pronom sujet [...], est appelé 'infinitif historique' ou 'infinitif de narration'. Il est très développé dans une langue littéraire de registre familier, mais très rare dans la langue parlée. Il présente, au cours d'un récit, une action comme la conséquence logique, mais réelle et rapide, de l'action précédente. Les actions exprimées par ce procédé sont surtout des mouvements, ou des réactions psychologiques » (GLLF, 2682).
} 


\section{L'infinitif substitut dans le récit}

\section{a) L'infinitif substitut marque une suite d'actions ou d'événements}

Gadet (1992 : 91) parle, pour le français populaire, d'«infinitifs juxtaposés au reste de la phrase, faisant suite à une série de temps du récit ».

[4] Je me suis retrouvé à zoner/ j’ai connu des copains/ (...) / ramassé par les flics/ repartir le matin/ refaire la manche/ remonter le soir (Gadet 1992 : 91).

On rencontre le même phénomène dans les parlers acadiens où les constructions infinitives sont souvent reliés par l'élément coordonnant pis.

[5] Pis là j'as té obligé de travailler dans les maisons aider les enfants pis essayer de ieux donner quoi-ce qui vouliont (NB - A, $8: 131-132)$.

[6] Il a dit aux hommes vite levez-vous. fait que les hommes s'a levé. et pis s'habiller. pis il ont mis les loups-marins à bord du canot. pis s'en ont été sus =a glace. prendre des barils des barils à la/ la grosse glace. pis quand il ont arrivé là la/ la glace a té tout' défait (IdlM, EN02, 387-390).

À ce propos, Wiesmath (2006: 84) constate :

«Au niveau de l'asyndète, on a pu aussi relever l'ellipse du pronom sujet dans l'une des séquences, le plus souvent dans la dernière. Sémantiquement, les verbes des deux séquences expriment des actions qui simplement se succèdent. C'est aussi le cas là où un infinitif remplace un verbe conjugué. Les marques du verbe fini (personne et temps) étant valables pour l'infinitif, il s'agit là d'un moyen syntaxique beaucoup plus intégratif servant à exprimer la simple progression du récit ».

L'infinitif substitut, qui, dans ces exemples, est précédé soit par (et) pis, soit par une pause, apparaît ainsi comme un moyen « économique » d'assurer la progression du récit ${ }^{10}$.

\section{b) L'infinitif substitut sert également à la description des routines ou d'actions et d'événements récurrents :}

[7] On commence d'abord par un shampooing, ensuite on les sèche en brossant et ensuite il y a la coupe. Suivant les chiens, les caniches une coupe, les fox une coupe et puis euh autrement les gros chiens, c'est juste un shampooing et puis les démêler, les démêler, les bobtails, les brillards, ça se démêle (Beeching, 10/15-19).

[8] Vous les netteyez comme i faut pis vous prenez des foies, pis de la farine et pis des ègnons pis mettre ensemble (Brasseur 1998 : 86).

\footnotetext{
${ }^{10}$ L'élément coordonnant pis peut éventuellement être remplacé par on bien, qui introduit ici une reformulation de la proposition: «je faisais plus la chasse aux chevreuils ou bien tuer des chevreuils » $(\mathrm{NB}-\mathrm{W}, 37)$.
} 
$V$ aleur sémantique et comportement syntaxique de l'infinitif substitut dans quelques corpus oraux

[9] Se lever, pis s'habiller, pis se partir, pis traverser à la Baie-Saint-Paul, pis après ça ben, arriver dans l'après-midi, à une autre marée qui revient, un autre (Seutin 1975 : 294).

[10] Ç’a été un bon cultivateur extra, pis un vive-la-joie, placoter d'un bord à l'autre, pis faire la mi-carême, pis chanter à l'église, chercher son trente-cinq cennes tous les matins, aller à l'église en bicycle (Seutin 1975 : 295).

[11] On le coupait par blocs et le fendre pis on faisait un feu pis le brûler à mesure (TN - B 2001: XLIX).

[12] ben nous autres on appelat ça la jornée des fous. Mais réellement y en a qui fait. des tours de fous des tours à hacher des arbres des/ des/. des/ des navets des arbres [...] pis. éparer ça tout le tour de la maison pis: ah c'est effrayant. changer les roues de charrette. pace qu'avant tout le monde avait des voitures vous savez des/ des chevaux. pis des grosses charrettes et des WAGONS et tout' ça. démancher des roues. pis aller l'amancher sus la/ sus la charrette du voisin pis changer là/ changer ça (IdlM, GC01, 1230-1248).

Brasseur (1998: 85) évoque la possibilité selon laquelle l'usage de l'infinitif serait «parfois aussi tout simplement entraîné par la présence d'une première forme verbale à l'infinitif dans la phrase précédente » (1998 : 85). Ceci pourrait être le cas de l'exemple [13] :

[13] Tu te levais le matin pis tu t'en allais faire ène marée de pêche. Pousser ton doury pis t'allais au large (TN - LC 029216).

Cet exemple soulève le problème de la graphie. À l'oral, les terminaisons de l'imparfait -ais, -ait et -aient peuvent éventuellement être assimilées aux infinitifs en -er. Le même argument pourrait être invoqué pour l'exemple [14] :

[14] $\mathrm{i}$ venaient/ $\mathrm{i}$ veniont une fois ba tous les mois une fois par mois euh : confesser i disaient la messe (NB - A, 9 : 124-125).

Dans les parlers acadiens qui connaissent la terminaison de l'imparfait en [a $]^{11}$ (v. ex. [11]: on appelat), les confusions possibles sont réduites. Comme dans le cas des infinitifs marquant une suite d'actions, nous trouvons cette construction après une pause ou bien précédé de et ou - plus souvent - pis (parfois suivi d'une pause, comme dans l'exemple [12]).

\section{La valeur finale de l'infinitif substitut}

L'infinitif substitut peut revêtir une valeur finale. Dans les occurrences que nous avons relevées, ces infinitifs sont systématiquement précédés d'une pause.

\footnotetext{
11 C’est le cas en Nouvelle-Écosse, aux Îles-de-la-Madeleine et à Terre-Neuve (v. Falkert 2007).
} 
Anika Falkert

[15] Quand tu avais cinquante cordes ben tous ces cinquante cennes là cinquante cordes ça te faisait un bon montant hein. payer toutes les dépenses $(\mathrm{NB}-\mathrm{A}$, $9: 46-47)$.

[16] On avait des COLEMAN STOVE. bouillir ça dessus (NB - W, 436).

[17] Les voisins se mettont ensemble dans un après midi. Ramasser peut-être dix douze balles de coton. Un après-midi. Ça menait là-là. De tous les bords (LOU - S, 215).

Wiesmath (2006 : 233) fait remarquer qu'en acadien,

«l'élément indiquant la valeur finale, la préposition pour, peut s'effacer. La tâche de préciser le rapport implicite de but à partir du contexte revient ainsi à l'auditeur ${ }^{12}$.

\section{L'infinitif substitut après (il) faut (que)}

Dans les parlers acadiens et en cadien, l'infinitif substitut remplace parfois les formes du subjonctif dans les énoncés introduits par (il) faut (que), " où l'on trouve souvent un infinitif remplaçant une deuxième forme verbale reliée à la première par pis » (Neumann-Holzschuh $2003:$ 74).

[18] Faulait j’alle dans le carré pis garocher du foin (NB - W, 79).

[19] C'était pareil pour la femme comme pour l'homme c'était encore pire t'étais pris $\mathrm{a}=\mathrm{ec}$ ène trâlée d'enfants des: huit dix enfants là à l'entour des jambes de même pis faulat qu'a faise à manger pis =ore racmoder le linge là coudre du linge qu'était/ mettre des pièces. ce tait pas/ aujourd'hui on jette ça dans le coin pis va y mettre ène autre paire de culottes sus le dos là (IdlM, PL02, 896-899).

Les exemples suivants ne représentent pas le même cas de figure dans la mesure où l'infinitif substitut est coordonné à une forme verbale soit à l'indicatif présent (exemples [20] et [21], soit au conditionnel ([22]).

[20] Ça fait un gros. Arbre faut tu vas puis le couper (LOU - S, 55).

[21] Y en a qu'achetait pour cinq sous, pour dix sous et il fallait que tu en prends ça dans le bateau-debarcadère, il fallait que tu $\underline{\text { marches }}^{13}$ là-bas, aller dans xxx (Lou - LFDP, PC/TB, p.6).

[22] Faut/ faut je sors dessus la galerie pour la barrer. là faurrait je marcherais d'en bas et puis revenir (Lou - S, 65).

On distinguera alors deux niveaux de «simplification»dont témoignent les exemples [20] à [22]. La première étape serait le remplacement du subjonctif après (il)

\footnotetext{
12 Selon Wiesmath, il ne s'agit, dans ce cas, pas d'un infinitif substitut, mais d'une simple omission de pour.

13 Pour les verbes du $1^{\text {er }}$ groupe, les formes du subjonctif et de l'indicatif présent se confondent. V. aussi les exemples [23] à [25].
} 
faut (que) par l'indicatif présent ou le conditionnel, alors que la deuxième étape prévoit l'emploi d'une forme invariable qui est coordonnée au premier verbe de la proposition subordonnée :

$$
\begin{aligned}
& \text { (il) faut (que) [PRONOM + VERBE au subj.] pis [PRONOM + VERBE au subj.] } \\
& =>\text { (il) faut (que) [PRONOM + VERBE à l'indic. prés/ au cond.] pis [VERBE à } \\
& \text { l'infinitif]. }
\end{aligned}
$$

Les énoncés [23] à [25] restent ambigus puisque «(faulut) je plonge », «(faulait $q^{\prime}$ ') i se mettent» et "(faulait que) t'arranges » peuvent être interprétés comme des formes à l'indicatif présent ou au subjonctif.

[23] Faulut je plonge en bas. Des barils. Puis déprendre le fil qu'était pris sur le baril (LOU - S, 115).

[24] Faulait qu'i se mettent de genoux pis laver ça avec une brousse (NB - W, 93).

[25] Tu restais pas couché $j=$ qu'à midi là faulait se lever de bonne heure. on coupait du bois surtout quand i donnait du mauvais temps là faulait que t'arranges tu coupes tout le bois quand i faisait beau pis aller chercher du foin dans les dunes (IdlM, PL02, 673-676).

\section{L'infinitif substitut dans les propositions hypothétiques}

L'infinitif substitut peut figurer également dans un énoncé introduit par $s i$ dans les hypothèses envisagées comme contraires à l'état des choses ou portant sur un fait passé qui s'est révélé faux.

[26] Si tout le monde faisait sa part / commencer à nettoyer chez eux pis faire / faire tout ce qu'ils peuvent / eux autres même / faire attention quand y vont au lac / pis pas jeter les affaires dans l'eau (Estrie, P261, L2-5).

[27] Sans ça, s'il avait eu en resté deboute, pis se branler (= s'agiter), ça lui aurait passé par-dessus la tête (Seutin 1975 : 296).

On remarquera que l'infinitif substitut est toujours précédé d'une forme verbale conjuguée, fonctionnant comme point de repère et qui guide le récepteur dans l'interprétation de l'énoncé. Cependant, on observe en français québécois une certaine autonomisation de l'infinitif dans les constructions hypothétiques où, comme le montrent les exemples [28a] et [28b], il remplace $s i+$ verbe conjugué : "L'infinitif concurrence $[s i+$ verbe à l'imparfait $]$ pour exprimer une condition non réalisée » (Léard 1995 : 192).

[28] a. Avoir une auto, jirais, certain (Léard 1995 : 192).

b. Avoir neigé, je serais pas venue (Léard 1995 : 192).

Seutin (1975) avait déjà signalé l'existence de cet «infinitif à valeur hypothétique » dans le parler de l'Île-aux-Coudres (Québec).

[29] Avoir su ça, je serais pas venu (Seutin 1975 : 296). 
Ces structures infinitives hypothétiques sont également attestées en francoontarien (qui est largement apparenté au français québécois) :

[30] Dire m'as avoir des millions là, ça serait pas pour moi, j'donnerais ça à mes filles (Mougeon 1995 : 114).

[31] Maintenant, j'pense que recommencer, j'frais une garde-malade (Mougeon 1995 : 114).

Il faut préciser que nous n'avons pas trouvé d'exemples de cette construction dans les corpus que nous avons consultés ${ }^{14}$.

\section{Conclusion : L'infinitif substitut - une construction polyfonctionnelle}

L'infinitif substitut comme substitut d'un temps du récit et du subjonctif après (il) faut (que) affiche une diffusion assez large dans les variétés de français. Par contre, son emploi dans les constructions hypothétiques ne semble attesté qu’au Québec (v. Seutin 1975 et Léard 1995) et en Ontario (v. Mougeon 1995). La fréquence d'occurrence selon les temps verbaux (présent, imparfait, passé composé) et les modes (conditionnel, subjonctif) que l'infinitif est censé remplacer est forcément inégale puisque les corpus sont constitués principalement de récits (de vie $)^{15}$ dans lesquels les événements passés jouent un rôle prépondérant.

Nous avons évoqué plus haut la tendance à l'invariance qui caractérise les évolutions en français parlé. L'infinitif substitut s'avère, à cet égard, comme une construction polyfonctionnelle qui, au moins dans les variétés hors de France, semble bien ancrée dans les échanges verbaux. Malgré l'indépendance syntaxique relative de l'infinitif substitut qui se manifeste également au niveau suprasegmental (on pensera aux exemples où, après une pause, le fil du récit est repris par l'infinitif), le lien sémantique reste fort. Pour illustrer cet aspect, il nous semble prudent de distinguer entre l'infinitif substitut proprement dit et les énoncés « intermédiaires » (exemples [32] et [33]) dans lesquels le rapport entre la forme verbale conjuguée et l'infinitif est encore visible :

[32] J'aimais à faire du patin / j'allais patiner beaucoup / pis faire un peu de ski (Estrie, P112/L27-28).

[33] J'aime bien que la vaiselle soit faite, que ce soit rangé et puis aller à la plage à une heure plutôt que de traîner et voir tout en désordre. (Beeching 7/118119).

Dans les deux cas, on pourrait évoquer une dépendance de l'infinitif de aimer (à) faire qch. Ceci permettrait d'émettre l'hypothèse selon laquelle l'infinitif substitut serait né d'une extension de ces contextes d'emploi.

\footnotetext{
${ }^{14} \mathrm{~V}$. à ce sujet les notes $5,6,7$ et 8.

15 Pour une discussion d'éventuelles spécificités syntaxiques des différents 'genres' discursifs v. Cappeau 2005.
} 
Valeur sémantique et comportement syntaxique de l'infinitif substitut dans quelques corpus oraux

L'ellipse du pronom personnel et l'infinitif substitut peuvent être interprétés comme deux phénomènes qui illustrent le principe d'économie ${ }^{16}$. Si l'infinitif dans les énoncés « intermédiaires » peut être interprété comme un complément du verbe, les liens syntaxiques sont souvent très lâches. La dépendance syntaxique des infinitifs dans les exemples [32] et [33] se distingue de la dépendance sémantique qui relie les infinitifs substituts proprement dits aux formes verbales conjuguées. L'infinitif substitut illustre ainsi la tendance à éviter la redondance au niveau du marquage du sujet dans les rapports de coordination et à réduire le paradigme à une seule forme invariable, précédée dans la plupart des cas de puis/pis.

\section{RÉFÉRENCES BIBLIOGRAPHIQUES}

ARrighI, L. (2005), Étude morphosyntaxique du français parlé en Acadie. Une approche de la variation et $d u$ changement linguistique en français. Thèse de doctorat, Université d'Avignon.

Beauchemin, N./Martel, P./Theoret, M. (1973-81), Échantillon de textes libres. Sherbrooke : Université de Sherbrooke.

Berrendonner, A. (1990), « Pour une macro-syntaxe », Travaux de linguistique, 21, pp. 25-36.

Blanche-Benveniste, C. (2000), Approches de la langue parlée en français. Paris : Ophrys.

BLANCHE-BENVENISTE, C. et al. (1990), Le français parlé. Études grammaticales. Paris : CNRS.

BRASSEUR, P. (1998), «De l'ellipse du pronom personnel aux formes verbales non marquées dans les parlers acadiens ", in: Patrice Brasseur (éd.), Français d'Amérique. Variation, créolisation, normalisation. Avignon : CECAV, pp. 75-91.

Brasseur, P. (2001), Dictionnaire des régionalismes du français de Terre-Neuve. Tübingen: Niemeyer.

CAPPEAU, P. (2005), «Ce que l'oral nous a appris sur la syntaxe du français », in : Corpus oraux et diversité des approches, Lidil 31, pp. 157-177.

FALKeRT, A. (2006), « La mutation achevée du connecteur ça fait que dans le parler acadien des Îles-de-la-Madeleine », Revue canadienne de linguistique appliquée, 9 (2), pp. 39-53.

FALKERT, A. (2007), Le français acadien des Îles-de-la-Madeleine. Étude de la variation phonétique. 3 vol. Thèse de doctorat, Université d'Avignon/Université de Regensburg.

GADET, F. (1989), Le français ordinaire. Paris : Armand Colin.

GADET, F. (1992), Le français populaire. Paris : PUF.

GLLF: Grand Larousse de la langue française, sous la direction de Louis Guilbert, René Lagane et Georges Niobey (1971-78). 7 vol. Paris : Larousse.

\footnotetext{
${ }^{16}$ Nous avons décidé de ne pas aborder, dans le cadre de cet article, la question de savoir si le français est susceptible, en certaines de ses variétés, d'être une langue pro-drop. Nous renvoyons le lecteur intéressé par cette problématique à l'article de Neumann-Holzschuh/Brasseur/Wiesmath (2005) qui soulignent que l'ellipse du pronom sujet correspond à des valeurs stylistiques particulières et qu'elle n'affiche pas la même fréquence dans les différentes variétés.
} 
Anika Falkert

Grevisse, M. (2008), Le Bon Usage. 14e éd. (refondue par André Goosse). Paris/Louvain-laNeuve : Duculot.

LÉARD, J.-M. (1995), Grammaire québécoise d'aujourd'bui - Comprendre les québécismes. Montréal : Guérin.

LE Goffic, P. (2005), «La phrase 'revisitée'», Le Français Aujourd'bui, 148, pp. 55 - 64 [republication de 2001 : Le Français Aujourd'bui, 135, pp. 97-107].

Mougeon, F. (1995), Quel français parler? Initiation au français parlé au Canada et en France. Toronto : Éditions du Gref.

NeumanN-Holzschuh, I. (2003), «Les formes verbales invariables en créole: un cas de réanalyse », in : Sibylle Kriegel (dir.), Grammaticalisation et réanalyse. Approches de la variation créole et française. Paris : CNRS, pp. 69-86.

Neumann-Holzschuh, I./Brasseur, P./Wiesmath, R. (2005), «Le français acadien au Canada et en Louisiane: affinités et divergences ", in: Albert Valdman/Julie Auger/Deborah Piston-Hatlen (éds.), Le français en Amérique du Nord. État présent. Québec : Presses de l’Université Laval, pp. 479-504.

ReMACLE, L. (1952-60), Syntaxe du parler wallon de la Gleize. 3 vol. Paris : Les Belles Lettres.

SEUTIN, Émile (1975), Description grammaticale du parler de l'Île-aux-Coudres. Montréal : Presses de l’Université de Montréal.

STÄBLER, C. (1995), La vie dans le temps et asteur. Ein Korpus von Gesprächen mit Cadiens in Louisiana. Tübingen : Narr.

Valdman, A. et al. (2004) (dir.), A la découverte du français cadien à travers la parole / Discovering Cajun French through the spoken word (CD-ROM). Bloomington : Indiana University Creole Institute.

Wiesmath, R. (2006), Le français acadien. Analyse syntaxique d'un corpus oral recueilli an NouveauBrunswick / Canada. Paris: L'Harmattan. 